\title{
Analysis of Relativistic Particle Orbit in a Transversely Focused High-Power Laser Field by Using the Noncanonical Lie Perturbation Method
}

\author{
Natsumi IWATA, Kenji IMADERA and Yasuaki KISHIMOTO \\ Department of Fundamental Energy Science, Graduate School of Energy Science, Kyoto University, \\ Gokasho, Uji, Kyoto 611-0011, Japan
}

(Received 19 July 2010 / Accepted 21 July 2010)

\begin{abstract}
A methodology describing the particle motion in a spatially localized high-power laser field in the relativistic regime is presented based on the noncanonical Lie perturbation method, which is comprehensive compared with those based on the conventional averaging method to the equation of motion. We successfully derived the relativistic ponderomotive force in a laser field with a radial intensity gradient and the corresponding particle dynamics up to the first order with respect to the expansion parameter $\varepsilon \sim \lambda / L$ (= laser wavelength/scale length of the transverse laser amplitude). The longitudinal motion was found to be secular and characterized by a growing oscillation exhibiting a cubic dependence on the laser amplitude.
\end{abstract}

(C) 2010 The Japan Society of Plasma Science and Nuclear Fusion Research

Keywords: noncanonical perturbation method, Lie transformation, high intensity laser field, ponderomotive force

DOI: $10.1585 /$ pfr.5.028

The development of ultra-short pulse high-power lasers with intensities of $10^{18-22} \mathrm{~W} / \mathrm{cm}^{2}$ has opened up various applications such as compact accelerators, fast ignition-based laser fusion and high-intensity $\mathrm{X}$ ray/neutron sources [1]. Recently, innovative ideas aiming at higher intensities of $10^{23-26} \mathrm{~W} / \mathrm{cm}^{2}$, where not only electrons, but also ions can exhibit relativistic characteristics, have been proposed. These ideas may lead to new scientific discoveries that have not been experienced in laboratories [2]. In order to realize such high intensities, a further reduction in the pulse width and/or the spot size is necessary. In such spatially localized laser fields, the ponderomotive force (light pressure) exists inevitably and plays an essential role $[3,4]$. The ponderomotive force has traditionally been derived by applying the averaging method directly to the equation of motion using the spatio-temporal scale separation. However, the method is not so prospective in capturing the particle motion precisely, especially in the tightly focused nonlinear relativistic regime where the higher-order terms, such as the curvature of the laser field amplitude, play an important role.

The Hamiltonian perturbation theory employing noncanonical variables and the Lie transformation has been investigated for analyzing the particle motion in electromagnetic fields [5]. The method has been successfully introduced as the gyro-kinetic formalism for describing magnetically confined fusion plasmas [6]. Kishimoto et al. applied the method for the first time to the analysis of a relativistic beam orbit in a free-electron laser and showed that the method is superior and powerful in describing the relativistic beam dynamics [7].

Motivated by these achievements, we extend this method to the analysis of the particle motion irradiated by spatially localized high-power lasers. As the first step, here, we derive the relativistic ponderomotive force and the related particle dynamics in a focused laser field.

We consider a particle with charge $q$ in a high intensity laser field propagating in the $z$-direction but localized in the transverse $x$-direction, which is expressed by the vector potential, $\boldsymbol{A}(x, \eta)=A_{x} \hat{\boldsymbol{e}}_{x}=A_{0}(x) \sin \eta \hat{\boldsymbol{e}}_{x}$, where $\eta \equiv \omega t-$ $k z$ is the phase. Here, we introduce an expansion parameter $\varepsilon \sim \lambda / L$, where $\lambda(=2 \pi / k)$ and $L\left(\equiv\left|\partial_{x} \log A_{0}\right|^{-1}\right)$ are the laser wavelength and the transverse scale length of the laser field amplitude, respectively. Using this parameter, we expand the amplitude of the vector potential around the initial particle position $x=x_{0}$ as

$A_{0}(x)=A_{0}\left(x_{0}\right)+\varepsilon \tilde{x} \partial_{x} A_{0}\left(x_{0}\right)+\varepsilon^{2} \frac{\tilde{x}^{2}}{2 !} \partial_{x}^{2} A_{0}\left(x_{0}\right)+\cdots$,

where $\tilde{x}=x-x_{0}$ and $\partial_{x} A_{0}\left(x_{0}\right)$ denotes $\partial A_{0}(x) /\left.\partial x\right|_{x=x_{0}}$.

Here, we perform the Hamiltonian perturbation analysis by using noncanonical variables and the Lie transformation. We introduce the extended phase space expressed by the canonical variables as $z^{\mu}=\left(t ; \boldsymbol{q}, \boldsymbol{p}_{c}\right)=$ $\left(t ; q_{x}, q_{y}, q_{z}, p_{c x}, p_{c y}, p_{c z}\right)$, where the time $t$ is the independent variable. The corresponding covariant vector is given by $\gamma_{\mu}=\left(-h ; \boldsymbol{p}_{c}, \mathbf{0}\right)$, where $h$ is the relativistic Hamiltonian expressed as 


$$
h\left(\boldsymbol{q}, \boldsymbol{p}_{c}, t\right)=\sqrt{m^{2} c^{4}+c^{2}\left(\boldsymbol{p}_{c}-\frac{q}{c} \boldsymbol{A}\right)^{2}} .
$$

In this paper, we use Latin indices that run from 1 to 6 whereas Greek from 0 to 6 . Using these notations, the variational principle from which the equations of motion can be derived is expressed as $\delta \int \gamma_{\mu} \mathrm{d} z^{\mu}=0$. The general transformation law from $\gamma_{\mu}$ to the new covariant vector $\Gamma_{\mu}$ under arbitrary coordinate transformation $z^{\mu} \rightarrow Z^{\mu}$ is given by $\Gamma_{\mu}=\gamma_{\nu} \partial z^{v} / \partial Z^{\mu}$.

As a preparatory transformation, we first introduce a noncanonical coordinate, $z^{\mu}=(t ; \boldsymbol{x}, \boldsymbol{p})=$ $\left(t ; x, y, z, p_{x}, p_{y}, p_{z}\right)$, where $\boldsymbol{p}=\boldsymbol{p}_{c}-q \boldsymbol{A} / c$ is the mechanical momentum and $\boldsymbol{x}=\boldsymbol{q}$. The corresponding covariant vector is then calculated as $\gamma_{\mu}=\left(-h ; p_{x}+\right.$ $\left.q A_{x}(x, z, t) / c, p_{y}, p_{z}, 0,0,0\right)$, where the Hamiltonian is expressed as $h=\sqrt{m^{2} c^{4}+c^{2} p^{2}}$. Note that the field $\boldsymbol{A}$ does not explicitly appear in the Hamiltonian but in the first component of $\gamma_{\mu}$, which simplifies the perturbation analysis.

Next, we introduce phase $\eta$ instead of the time $t$ as the independent variable in order to distinguish the secular motions from the whole particle dynamics. This procedure is also the noncanonical transformation by which the new coordinate $z^{\mu}$ and the new covariant vector $\gamma_{\mu}$ are obtained as

$$
\begin{aligned}
& z^{\mu}=\left(\eta ; x, y, z, p_{x}, p_{y}, p_{z}\right), \\
& \gamma_{\mu}=\left(-K ; p_{x}+q A_{x}(x, \eta) / c, p_{y}, p_{z}-k K, 0,0,0\right),
\end{aligned}
$$

where $K=\omega^{-1} \sqrt{m^{2} c^{4}+c^{2} \boldsymbol{p}^{2}}$ is the new Hamiltonian.

In this coordinate system, the unperturbed particle motion $z^{i(0)}$ is obtained as

$$
\begin{aligned}
& x^{(0)}=\frac{c a_{0}}{\omega} \cos \eta-\frac{c a_{0}}{\omega}+x_{0}, \\
& y^{(0)}=0, \\
& z^{(0)}=\frac{c a_{0}^{2}}{4 \omega}\left(\eta-\frac{1}{2} \sin 2 \eta\right), \\
& p_{x}^{(0)}=-m c a_{0} \sin \eta, \\
& p_{y}^{(0)}=0, \\
& p_{z}^{(0)}=\frac{m c a_{0}^{2}}{4}(1-\cos 2 \eta),
\end{aligned}
$$

under the initial condition $\left(\boldsymbol{x}_{0}, \boldsymbol{p}_{0}\right)=\left(x_{0}, 0,0,0,0,0\right)$ at $\eta=0$. Here, $a_{0} \equiv q A_{0}\left(x_{0}\right) / m c^{2}$ denotes the normalized laser amplitude. As is well known, the particle exhibits a figure-eight motion drifting in the direction of the laser propagation with momentum $m c a_{0}^{2} / 4[8] . \quad p_{z}^{(0)}$ in Eq. (10) can also be expressed as $p_{z}^{(0)}=m c\left(\gamma^{(0)}-1\right)$, where $\gamma^{(0)}\left(=\sqrt{1+\left(\boldsymbol{p}^{(0)} / m c\right)^{2}}\right)$ is the relativistic factor of the zeroth-order.

To investigate the secular motion originating from the gradient of the laser field, we transform the coordinate $z^{\mu}$ (Eq. (3)) to that of the oscillation-center $Z^{\mu}$ drifting with the momentum $\boldsymbol{p}=\hat{\boldsymbol{e}}_{z} m c a_{0}^{2} / 4$, where the relationship between the old and new coordinates is given by $z^{i}=Z^{i}+z^{i(0)}$.
Then, the new coordinate and the corresponding covariant vector are obtained as

$$
\begin{aligned}
Z^{\mu}= & (\eta ; X, Y, Z, U, V, W), \\
\Gamma_{\mu}= & \left(-\kappa ; U+m c\left(a_{0}(x)-a_{0}\right) \sin \eta, V,\right. \\
& \left.\quad W+\frac{m c a_{0}^{2}}{4}(1-\cos 2 \eta)-k K, 0,0,0\right)
\end{aligned}
$$

with $a_{0}(x) \equiv q A_{0}(x) / m c^{2}$. Here, $\kappa=K-\gamma_{1} \partial x / \partial \eta-\gamma_{3} \partial z / \partial \eta$ is the new Hamiltonian calculated using the relation between Eqs. (3) and (11), which yields

$$
\begin{aligned}
\kappa= & K+\frac{c a_{0}}{\omega}\left[\left(U+p_{x}^{(0)}+m c a_{0}(x) \sin \eta\right) \sin \eta\right. \\
& \left.-\frac{a_{0}}{4}\left(W+p_{z}^{(0)}-k K\right)(1-\cos 2 \eta)\right] .
\end{aligned}
$$

Note that the argument in Eqs. (12) and (13) is expressed by $Z^{\mu}$, e.g., $x=x(\eta, X)$. On the basis of these coordinate and covariant vector, we perform a perturbation analysis based on the expansion, Eq. (1). In the zeroth order, $a_{0}(x)$ in Eqs. (12) and (13) becomes $a_{0}$. The zeroth-order solution is then obtained as $Z^{(0) i}=(0,0,0,0,0,0)$.

To analyze the first-order motion by using the Lie perturbation method, we transform the coordinate to a new one, $Z^{\prime \mu}$, in which the first-order covariant vector can be simplified as $\Gamma_{\mu}^{(1)}=\left(\left\langle V^{(0) \mu} \Gamma_{\mu}^{(1)}\right\rangle ; \mathbf{0}, \mathbf{0}\right)$, where $\left\langle V^{(0) \mu} \Gamma_{\mu}^{(1)}\right\rangle=-\lambda a_{0}^{2} m c\left(X^{\prime}-c a_{0} / \omega\right) / 4 \pi L$. Here, $V^{(0) \mu}$ is the unperturbed flow vector defined by $V^{(0) 0}=1, V^{(0) i}\left(Z^{\mu}\right)=$ $\mathrm{d} Z^{(0) i} / \mathrm{d} Z^{0}$. Then, the new covariant vector up to the first order is obtained by $\Gamma_{\mu}^{\prime}=\Gamma_{\mu}^{(0)}+\varepsilon \Gamma_{\mu}^{\prime(1)}$. The oscillationcenter equations of the motion up to the first order are obtained as follows:

$$
\begin{aligned}
& \frac{\mathrm{d} \boldsymbol{X}^{\prime}}{\mathrm{d} \eta}=\frac{\boldsymbol{P}^{\prime}}{m \omega}, \\
& \frac{\mathrm{d} U^{\prime}}{\mathrm{d} \eta}=-\varepsilon \frac{\lambda}{2 \pi L} \frac{a_{0}^{2}}{2} m c, \\
& \frac{\mathrm{d} V^{\prime}}{\mathrm{d} \eta}=0, \\
& \frac{\mathrm{d} W^{\prime}}{\mathrm{d} \eta}=m c \frac{\mathrm{d} \gamma^{\prime}}{\mathrm{d} \eta}-\frac{a_{0}^{2}}{2} m c \sin 2 \eta,
\end{aligned}
$$

where $\boldsymbol{X}^{\prime}=\left(X^{\prime}, Y^{\prime}, Z^{\prime}\right), \boldsymbol{P}^{\prime}=\left(U^{\prime}, V^{\prime}, W^{\prime}\right)$, and $\gamma^{\prime}$ is the relativistic factor obtained from the momenta up to the first order, i.e., $\gamma^{\prime}=\sqrt{1+\left[\left(\boldsymbol{P}^{\prime}+\boldsymbol{p}^{(0)}\right) / m c\right]^{2}}$. Equation (15) expresses the ponderomotive force that is proportional to the first derivative of the laser amplitude. Note that since we have chosen not the time $t$ but the phase $\eta$ as the independent variable, $\omega / \gamma^{\prime}(=\mathrm{d} \eta / \mathrm{d} t)$ is multiplied with respect to Eq. (15) to obtain the conventional expression for the ponderomotive force [3,4]. It is also noted that the influence of the ponderomotive force in the $z$-direction can be seen in Eq. (17) in the term proportional to $\mathrm{d} \gamma^{\prime} / \mathrm{d} \eta$. Equation (17) can be solved using the solutions of $U^{\prime}$ and $V^{\prime}$ as

$$
W^{\prime}=\varepsilon \frac{\lambda}{2 \pi L} \frac{a_{0}^{3}}{2} m c \eta \sin \eta
$$


which represents the oscillation term, but its amplitude secularly grows in the direction of the laser propagation. This is ascribed to the $\boldsymbol{v} \times \boldsymbol{B}$ effect caused by the interaction between the secular motion due to the ponderomotive force (Eq. (15)) and the background oscillating laser magnetic field. This may play an important role, especially in the highly relativistic regime because this term is proportional to $a_{0}^{3}$.

The solutions for the initial oscillation-center coordinate, Eq. (11), are obtained from the backward transformation $Z^{\mu}=Z^{\prime \mu}-\varepsilon g^{(1) \mu}$, where $g^{(1) \mu}$ is the first-order generator of the Lie transformation [5]. For instance, the longitudinal momentum $W$ is given as

$$
W=W^{\prime}-\varepsilon \frac{\lambda}{2 \pi L} \frac{a_{0}^{3}}{2} m c\left[1-\cos 2 \eta-\frac{1}{4}(\cos \eta-\cos 3 \eta)\right] .
$$

The second and third terms on the right-hand side of Eq. (19) correspond to the reduction in the zeroth-order longitudinal velocity. This reduction originates from the fact that the particle feels a weaker field on average during one cycle of the oscillations than it would in a uniform field. This result is consistent with that obtained by applying the perturbation analysis directly to the equation of motion derived from Eqs. (3) and (4). However, such an approach does not keep the Hamiltonian structure which is rigorously kept in the present analysis. In this case, the general theorems of Hamiltonian mechanics can be applied.

In conclusion, we have derived the equation system describing the relativistic pondromotive force and the related particle dynamics due to a transversely localized laser field up to the order $\varepsilon \sim \lambda / L$. We found that the recoil of the ponderomotive force was included in the same order $\varepsilon$ in the direction of the laser propagation and was represented as a secular term exhibiting a growing oscillation with a cubic dependence on the normalized amplitude. This perturbation expansion is valid for $\varepsilon a_{0}<O(1)$. The remarkable feature of the present noncanonical Lie perturbation analysis is the systematic and rigorous determination of the higher-order terms. Here, we have only derived terms up to the first order, but it can be extended to the second order which may include the curvature effect of the laser field.

One of the authors, N. Iwata, appreciates Drs. J. Koga and Y. Fukuda in the Advanced Photon Research Center, Japan Atomic Energy Agency (JAEA) for their encouragement during the JAEA summer program (2009), which initiated the present research. This study was supported by a Grant-in-Aid from JSPS (No. 21340171).

[1] G.A. Mourou, T. Tajima and S.V. Bulanov, Rev. Mod. Phys. 78, 309 (2006).

[2] T. Tajima, Scientific Advisory Committee: Report on the ELI Science (2009).

[3] E.A. Startsev and C.J. McKinstrie, Phys. Rev. E 55, 7527 (1996).

[4] P. Gibbon, Short Pulse Laser Interactions with Matter (Imperial College Press, London, 2005) p.36.

[5] J.R. Cary and R.G. Littlejohn, Ann. Phys. 151, 1 (1983).

[6] A.J. Brizard and T.S. Hahm, Rev. Mod. Phys. 79, 421 (2007).

[7] Y. Kishimoto, S. Tokuda and K. Sakamoto, Phys. Plasmas 2, 1316 (1995).

[8] E.S. Sarachik and G.T. Schappert, Phys. Rev. D 1, 2738 (1970). 